\title{
Preparation of $2,3,6,2^{\prime}, 3^{\prime}, 4^{\prime}, 6^{\prime}$-hepta- $O$-acetyl- maltose/cellobiose by enzymatic hydrolysis of maltose/cellobiose octaacetate
}

\author{
Geok-Toh Ong, Kung-Yao Chang, Shih-Hsiung Wu *, \\ Kung-Tsung Wang \\ Institute of Biological Chemistry, Academia Sinica and Graduate Institute of Biochemical Sciences, \\ National Taiwan University, P.O. Box 23-106, Taipei, Taiwan
}

Received 17 March 1994; accepted in revised form 5 July 1994

Keywords: Enzymatic deacetylation; Anomeric $O$-deacetylation; Lipase deacetylation; Protease deacetylation; Alcalase deacetylation; Maltose hepta-acetate; Cellobiose hepta-acetate

Partially protected monosaccharides with free anomeric hydroxyl groups are useful in the synthesis of oligosaccharides [1-3]. A number of chemical methods are reported to remove the anomeric acyl group of peracylated carbohydrates [4-6], providing a useful route to partially protected saccharides $[7,8]$. Enzymatic reactions have also been used for this purpose [9-23]. According to previous reports, primary acetyl esters in peracetylated sucrose are the most reactive sites for enzymatic hydrolysis [19-22], whereas the anomeric position in peracylated monosaccharides, including furanoses and pyranoses, is the most reactive site [23]. Fink and Hay reported that maltose and cellobiose octacetates ( 1 and 2) were hydrolyzed by wheat germ lipase with low regioselectivity and a mixture of products was obtained [9]. In our work, compounds 1 and 2 were hydrolyzed by various hydrolytic enzymes exclusively at the anomeric position.

More than ten enzymes were examined for the hydrolysis of 1 and 2; the results are listed in Tables 1 and 2. All enzymes tested preferentially cleaved the anomeric esters (Scheme 1). Among the enzymes, lipase AP-6 had the greatest rate of hydrolysis toward both substrates; protease II, protease $\mathrm{N}$, and lipase $\mathrm{N}$ had moderate rates; lipase $\mathrm{P}$, lipase $\mathrm{AK}$, lipase GC, and lipase CE showed only a small rate. Lipase OF, lipase FAP-15, and alcalase had a moderate rate toward $\mathbf{1}$, but a small rate toward 2 .

* Corresponding author. 
Table 1

Hydrolysis of maltose octaacetate by various enzymes

\begin{tabular}{llcr}
\hline Enzymes & Reaction solution & Reaction time $(\mathbf{h})$ & Yield $(\%)$ \\
\hline Lipase AP-6 & phosphate buffer & 1.5 & 98 \\
& $4: 1$ phosphate buffer-EtOH & 1.5 & 98 \\
Lipase OF & phosphate buffer & 96 & 90 \\
Lipase MY & phosphate buffer & 168 & $<20$ \\
Lipase P & phosphate buffer & 168 & $<20$ \\
Lipase N & phosphate buffer & 24 & 96 \\
Lipase AK & phosphate buffer & 168 & $<20$ \\
Protease II & $4: 1$ phosphate buffer-DMF & 8 & 90 \\
Alcalase & phosphate buffer & 48 & 70 \\
Protease N & $4: 1$ phosphate buffer-DMF & 24 & 85 \\
Lipase FAP-15 & phosphate buffer & 120 & 80 \\
Lipase AY 30 & phosphate buffer & 168 & $<20$ \\
Lipase CE & phosphate buffer & 168 & $<20$ \\
Lipase GC & phosphate buffer & 168 & $<20$ \\
PPL & phosphate buffer & 120 & $<20$ \\
\hline
\end{tabular}

The products obtained from hydrolysis of 1 by various enzymes showed two peaks in HPLC. After NMR analysis, two products were identified as the $\alpha$ and $\beta$ forms of $2,3,6,2^{\prime}, 3^{\prime}, 4^{\prime}, 6^{\prime}$-hepta- $O$-acetyl-maltose ( $3 \boldsymbol{\alpha}$ and $\mathbf{3} \boldsymbol{\beta}$ ) that interconverted through mutarotation. In order to demonstrate further the enzymatic cleavage at $\mathrm{C}-1$, we reacted the mixture of products $(3 \boldsymbol{\alpha}$ and $\mathbf{3} \boldsymbol{\beta})$ with tert-butyldimethylsilyl chloride in pyridine, resulting in tertbutyldimethylsilyl $2,3,6,2^{\prime}, 3^{\prime}, 4^{\prime}, 6^{\prime}$-hepta- $O$-acetyl- $\beta$-maltoside (5) (Scheme $1 \mathrm{a}$ ). This selectivity for the formation of the $\beta$ anomer is unexpected and may be ascribed to a steric effect.

Table 2

Hydrolysis of cellobiose octaacetate by various enzymes

\begin{tabular}{llcr}
\hline Enzymes & Reaction solution & Reaction time (h) & Yield (\%) \\
\hline Lipase AP-6 & phosphate buffer & 2.5 & 98 \\
& $4: 1$ phosphate buffer-EtOH & 2.5 & 98 \\
Lipase OF & phosphate buffer & 168 & $<20$ \\
Lipase MY & phosphate buffer & 168 & $<20$ \\
Lipase P & phosphate buffer & 168 & $<20$ \\
Lipase N & phosphate buffer & 168 & 90 \\
Lipase AK & phosphate buffer & 168 & $<20$ \\
Protease II & $4: 1$ phosphate buffer-DMF & 24 & 90 \\
Alcalase & phosphate buffer & 168 & $<20$ \\
Protease N & $4: 1$ phosphate buffer-DMF & 72 & 90 \\
Lipase FAP-15 & phosphate buffer & 168 & $<20$ \\
Lipase AY 30 & phosphate buffer & 168 & $<20$ \\
Lipase CE & phosphate buffer & 168 & $<20$ \\
Lipase GC & phosphate buffer & 168 & $<20$ \\
PPL & phosphate buffer & 168 & $<20$ \\
\hline
\end{tabular}



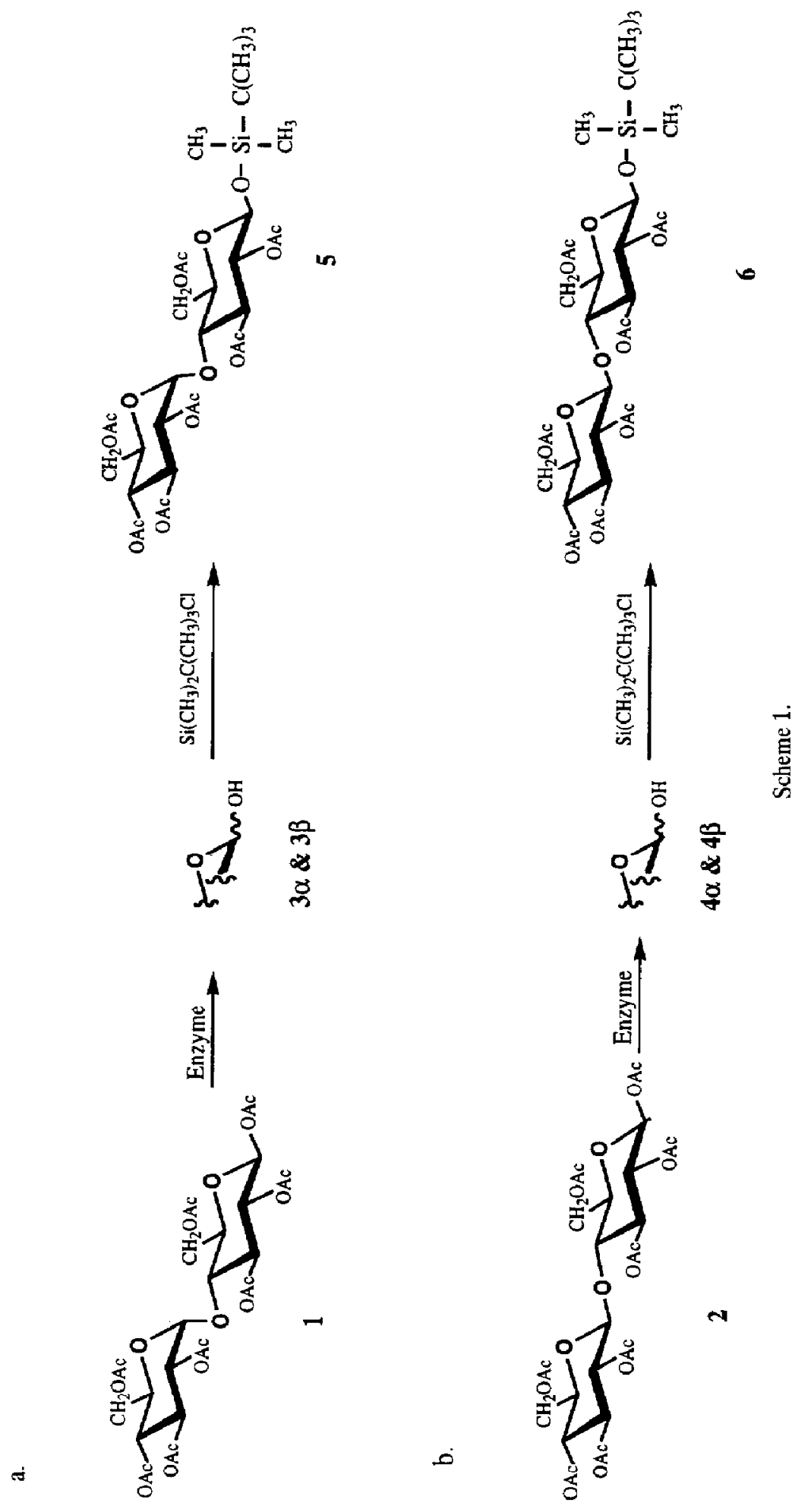
Table 3

${ }^{1} \mathrm{H}$ NMR chemical shifts of $1,3 \boldsymbol{\alpha}, 3 \boldsymbol{\beta}$, and 5

\begin{tabular}{|c|c|c|c|c|}
\hline & 1 & $3 \alpha$ & $3 \beta$ & 5 \\
\hline H-1 & $5.67\left(\mathrm{t}, J_{1,2} 8.0\right)$ & $5.27-5.35$ & $4.66-4.75$ & $4.72-4.76$ \\
\hline $\mathrm{H}-2$ & $4.89\left(\mathrm{dd}, J_{2,3} 8.6\right)$ & $4.72\left(\mathrm{dd}, J_{2,3} 9.7\right)$ & $4.66-4.75$ & $4.72-4.76$ \\
\hline $\mathrm{H}-\mathbf{3}$ & $5.22\left(\mathrm{t}, J_{3,4} 8.7\right)$ & $5.52\left(t, J_{3,4} 9.4\right)$ & $5.22\left(t, J_{3,4} 9.0\right)$ & $5.21\left(\mathrm{t}, J_{3,4} 9.0\right)$ \\
\hline $\mathrm{H}-4$ & $3.96-3.99$ & $3.94\left(t, I_{4,5} 9.9\right)$ & $3.94\left(\mathrm{t}, J_{4.5} 9.9\right)$ & $3.94\left(\mathrm{t}, J_{1,5} 9.2\right)$ \\
\hline $\mathrm{H}-5$ & $3.75-3.78(\mathrm{~m})$ & $4.14-4.21(\mathrm{~m})$ & $3.67-3.71(\mathrm{~m})$ & $3.64-3.70(\mathrm{~m})$ \\
\hline H-6a & $4.38\left(\mathrm{dd}, J_{5,6 \mathrm{a}} 2.3\right)$ & $4.43\left(\mathrm{dd}, J_{6 \mathrm{a}, 6 \mathrm{~b}} 11.3\right)$ & $4.43\left(\mathrm{dd}, J_{6 \mathrm{a}, 6 \mathrm{~b}} 11.3\right)$ & $4.43\left(\mathrm{dd}, J_{5,6 \mathrm{a}} 2.6\right)$ \\
\hline $\mathrm{H}-6 \mathrm{~b}$ & $\begin{array}{l}4.16\left(\mathrm{dd}, J_{5,6 \mathrm{~b}} 5.4\right. \\
\left.J_{6 \mathrm{a}, 6 \mathrm{~b}} 12.3\right)\end{array}$ & $4.14-4.21$ & $4.14-4.21$ & $\begin{array}{l}4.17\left(\mathrm{dd}, J_{5,6 \mathrm{~b}} 5.3,\right. \\
\left.J_{6 \mathrm{a}, 6 \mathrm{~b}} 11.9\right)\end{array}$ \\
\hline $\mathrm{H}-1^{\prime}$ & $5.33\left(\mathrm{~d}, J_{1,2}, 4.0\right)$ & $5.37\left(\mathrm{~d}, J_{1,2,2} 3.6\right)$ & $5.37\left(\mathrm{~d}, J_{1 r, 2 r} 3.9\right)$ & $5.38\left(\mathrm{~d}, J_{1,2}, 4.0\right)$ \\
\hline $\mathrm{H}-2^{\prime}$ & $4.78\left(\mathrm{dd}, J_{2,3}, 10.5\right)$ & $4.72\left(\mathrm{dd}, J_{2,3}, 10.5\right)$ & $4.80\left(\mathrm{dd}, J_{2,3,3}, 10.5\right)$ & $4.81\left(\mathrm{dd}, J_{2 r, 3}, 10.5\right)$ \\
\hline $\mathrm{H}-3^{\prime}$ & $5.27\left(t, J_{3,4}, 9.7\right)$ & $5.27-5.35$ & $5.27-5.35$ & $5.32\left(\mathrm{t}, J_{3,4}, 10.1\right)$ \\
\hline $\mathbf{H}-4^{\prime}$ & $4.97\left(\mathrm{t}, J_{4,5}, 9.9\right)$ & $5.00\left(t, J_{4,5}, 9.8\right)$ & $4.99\left(\mathrm{t}, J_{4,5}, 9.8\right)$ & $5.01\left(t, J_{4 f, 5}, 9.9\right)$ \\
\hline H-5 & $3.85-3.88(\mathrm{~m})$ & $3.90-3.97(\mathrm{~m})$ & $3.90-3.97(\mathrm{~m})$ & $3.91-3.97(\mathrm{~m})$ \\
\hline$H-6^{\prime} a$ & $4.14-4.18$ & $4.14-4.21$ & $4.14-4.21$ & $4.23\left(\mathrm{dd}, J_{5 s, 6, \mathrm{a}} 4.0\right)$ \\
\hline$H-6^{\prime} b$ & $3.96 \quad 3.99$ & 4.024 .04 & $4.02 \cdot 4.04$ & $\begin{array}{l}4.02\left(\mathrm{dd}, J_{3 x, 0, \mathrm{~b}} 1.9\right. \\
\left.J_{6, \mathrm{a}, 6, \mathrm{~b}} 12.5\right)\end{array}$ \\
\hline $\mathrm{COOCH}_{3}$ & $1.92-2.06(\mathrm{~m})$ & $1.94-2.11(\mathrm{~m})$ & $1.94-2.11(\mathrm{~m})$ & $1.98-2.10(\mathrm{~m})$ \\
\hline $\mathrm{SiC}\left(\mathrm{CH}_{3}\right)_{3}$ & & & & $0.84-0.88(\mathrm{~m})$ \\
\hline $\mathrm{Si}\left(\mathrm{CH}_{3}\right)_{2}$ & & & & $0.07-0.08(\mathrm{~m})$ \\
\hline
\end{tabular}

Two products were also obtained from hydrolysis of 2 with various enzymes. Using the same methods as described above, we identified them as the $\alpha$ and $\beta$ forms of $2,3,6,2^{\prime}, 3^{\prime}, 4^{\prime}, 6^{\prime}$-hepta- $O$-acetyl-cellobiose $(4 \alpha$ and $4 \beta)$ tert-butyldimethylsilyl $2,3,6,2^{\prime}, 3^{\prime}, 4^{\prime}, 6^{\prime}$-hepta- $O$-acetyl- $\beta$-cellobioside (6) was formed by reaction with tert-butylchlorodimethylsilane (Scheme $1 \mathrm{~b}$ ).

The structures of $3 \boldsymbol{\alpha}$ and $3 \boldsymbol{\beta}$ were determined mainly by ${ }^{1} \mathrm{H}-{ }^{1} \mathrm{H}$ COSY and ${ }^{1} \mathrm{H}-{ }^{13} \mathrm{C}$ HMQC (Heteronuclear Multiple Quantum Correlation) spectra. The chemical shifts of 1 , $\mathbf{3 \alpha}, \mathbf{3 \beta}$, and 5 are listed in Tables 3 and 4 . The chemical shift of $\mathrm{H}-1$ in $\mathbf{1}$, assigned at 5.67 $\mathrm{ppm}$, shifted upfield to $5.27-5.35 \mathrm{ppm}$ in $3 \boldsymbol{\alpha}$ and to $4.66-4.75 \mathrm{ppm}$ in $3 \boldsymbol{\beta}$ after deacetylation. These results indicate that the deacylation occurred at the anomeric site. The signals of $\mathbf{H}$ 3 and $\mathrm{H}-5$ in $3 \beta$ assigned at 5.22 and $3.67-3.71 \mathrm{ppm}$ are the same as those of 1 ; while the signals of the same two protons in $3 \alpha$ were shifted downfield to 5.52 and $4.14-4.21 \mathrm{ppm}$. The ${ }^{13} \mathrm{C}$ chemical shifts of $\mathrm{C}-1, \mathrm{C}-2, \mathrm{C}-3$, and $\mathrm{C}-5$ in $3 \boldsymbol{\beta}$ were found more downfield than those of $3 \alpha$. According to the intensity of ${ }^{1} \mathrm{H}$ NMR signals and the area of the HPLC profile, the ratio of $3 \boldsymbol{\alpha}$ to $3 \boldsymbol{\beta}$ was estimated to be $2.5: 1$. NMR data of 5 showed similar chemical shifts to those of $\mathbf{3 \beta}$ and the structure of $\mathbf{5}$ was identified to be tert-butyldimethylsilyl $2,3,6,2^{\prime}, 3^{\prime}, 4^{\prime}, 6^{\prime}$-hepta- $O$-acetyl- $\beta$-maltoside.

The structures of $4 \alpha, 4 \beta$, and 6 were determined and the interpretation and assignments of the spectra were similar to those of the maltose derivatives described above (Tables 5 and 6).

These results indicate that anomeric esters of 1 and 2 are the most reactive in the enzymatic reaction regardless of an $\alpha$ or $\beta$ glycosidic linkage between two glucosyl moieties. Enzy- 
Table 4

${ }^{13} \mathrm{C}$ NMR chemical shifts of $1,3 \alpha, 3 \beta$, and 5

\begin{tabular}{|c|c|c|c|c|}
\hline & 1 & $3 \alpha$ & $\mathbf{3} \boldsymbol{\beta}$ & 5 \\
\hline$C-1$ & 91.15 & 89.76 & 94.66 & 95.23 \\
\hline $\mathrm{C}-2$ & 70.83 & 71.53 & 73.56 & 74.01 \\
\hline $\mathrm{C}-3$ & 75.10 & 72.23 & 74.87 & 75.42 \\
\hline C-4 & 72.36 & 72.52 & 72.52 & 73.09 \\
\hline $\mathrm{C}-5$ & 72.88 & 67.46 & 72.52 & 72.12 \\
\hline C-6 & 62.42 & 62.74 & 62.74 & 63.02 \\
\hline$C-1^{\prime}$ & 95.61 & 95.36 & 95.36 & 95.49 \\
\hline $\mathrm{C}-2^{\prime}$ & 69.91 & 69.90 & 69.90 & 69.97 \\
\hline$C-3^{\prime}$ & 69.19 & 69.25 & 69.25 & 69.35 \\
\hline $\mathrm{C}-4^{\prime}$ & 67.87 & 67.88 & 67.88 & 68.04 \\
\hline$C-5^{\prime}$ & 68.47 & 68.25 & 68.25 & 68.51 \\
\hline$C-6^{\prime}$ & 61.35 & 61.30 & 61.30 & 61.60 \\
\hline $\mathrm{C}-\mathrm{COCH}_{3}$ & $168.66-170.43$ & $169.44-170.61$ & $169.44-170.61$ & $169.44-170.53$ \\
\hline $\mathrm{C}-\mathrm{COCH}_{3}$ & $20.41-2073$ & $20.48-20.74$ & $20.48-20.74$ & $20.60-20.94$ \\
\hline $\mathrm{Si}-C\left(\mathrm{CH}_{3}\right)_{3}$ & & & & 17.84 \\
\hline $\mathrm{Si}-\mathrm{C}\left(\mathrm{CH}_{3}\right)_{3}$ & & & & 25.40 \\
\hline \multirow[t]{2}{*}{$\mathrm{Si}-\left(\mathrm{CH}_{3}\right)_{2}$} & & & & -4.36 \\
\hline & & & & -5.47 \\
\hline
\end{tabular}

matic deacetylation then provides an efficient way to prepare $2,3,6,2^{\prime}, 3^{\prime}, 4^{\prime}, 6^{\prime}$-hepta- $O$ acetyl-maltose/cellobiose in high yield on a scale of up to $10 \mathrm{~g}$.

Table 5

${ }^{1} \mathrm{H}$ NMR chemical shifts of $2,4 \alpha, 4 \beta$, and 6

\begin{tabular}{|c|c|c|c|c|}
\hline & 2 & $4 \alpha$ & $4 \beta$ & 6 \\
\hline H-1 & $5.61\left(\mathrm{~d}, J_{1,2} 8.3\right)$ & $5.34\left(\mathrm{~d}, J_{1,2} 3.6\right)$ & $4.72\left(\mathrm{~d}, J_{1,2} 5.8\right)$ & $4.65\left(\mathrm{~d}, J_{1,2} 7.6\right)$ \\
\hline $\mathrm{H}-2$ & $4.98\left(\mathrm{dd}, J_{2,3} 9.4\right)$ & $4.77-4.82$ & $4.77-4.82$ & $4.81\left(\mathrm{dd}, J_{2,3} 9.7\right)$ \\
\hline $\mathrm{H}-3$ & $5.17\left(t, J_{3,4} 9.1\right)$ & $5.48\left(t, J_{3,4} 9.7\right)$ & $5.20\left(\mathrm{t}, J_{3,4} 9.0\right)$ & $5.12\left(t, J_{3,4} 9.7\right)$ \\
\hline $\mathrm{H}-4$ & $4.76\left(\mathrm{t}, J_{4,5} 9.2\right)$ & $3.72\left(t, J_{4,5} 9.3\right)$ & $3.77\left(\mathrm{t}, J_{4,5} 9.8\right)$ & $3.68\left(\mathrm{t}, J_{4,5} 9.3\right)$ \\
\hline $\mathrm{H}-5$ & $3.67-3.72(\mathrm{~m})$ & $4.10-4.20(\mathrm{~m})$ & $3.60-3.67(\mathrm{~m})$ & $3.53-3.66(\mathrm{~m})$ \\
\hline H-6a & $4.42\left(\mathrm{dd}, J_{5,6 \mathrm{a}} 1.6\right)$ & $4.47-4.51$ & $4.47-4.51$ & $4.41-4.43$ \\
\hline $\mathrm{H}-6 \mathrm{~b}$ & $\begin{array}{l}4.06\left(\mathrm{dd}, J_{5.6 \mathrm{~b}} 4.7\right. \\
\left.J_{6 \mathrm{a}, 6 \mathrm{~b}} 12.1\right)\end{array}$ & $3.98-4.20$ & $3.98-4.20$ & $4.04\left(\mathrm{dd}, J_{5,6 \mathrm{~b}} 6.0\right)$ \\
\hline $\mathrm{H}-1^{\prime}$ & $4.60\left(\mathrm{~d}, J_{1,2} 7.9\right)$ & $4.52-4.53$ & $4.52-4.53$ & 4.71 \\
\hline $\mathrm{H}-2^{\prime}$ & $4.86\left(\mathrm{t}, J_{2,3}, 8.5\right)$ & $4.94\left(t, J_{2 r, 3}, 8.6\right)$ & $4.94\left(\mathrm{t}, J_{2 r, 3,}, 8.6\right)$ & $4.88\left(\mathrm{t}, J_{2 r, 3}, 8.5\right)$ \\
\hline $\mathrm{H}-3^{\prime}$ & $5.08\left(\mathrm{t}, J_{3,4}, 9.2\right)$ & $5.17\left(\mathrm{t}, J_{3,4}, 9.2\right)$ & $5.17\left(\mathrm{t}, J_{3,4,}, 9.2\right)$ & $5.20\left(\mathrm{t}, J_{3 r, 4}, 9.3\right)$ \\
\hline $\mathrm{H}-4^{\prime}$ & $5.01\left(\mathrm{t}, J_{4,5}, 9.5\right)$ & $5.04\left(\mathrm{t}, J_{4,5}, 9.3\right)$ & $5.04\left(\mathrm{t}, J_{4,5}, 9.3\right)$ & $5.01\left(\mathrm{t}, J_{4,5}, 9.2\right)$ \\
\hline $\mathrm{H}-5^{\prime}$ & $3.58-3.64(\mathrm{~m})$ & $3.60-3.67(\mathrm{~m})$ & $3.60-3.67(\mathrm{~m})$ & $3.53-3.66(\mathrm{~m})$ \\
\hline$H-6^{\prime} a$ & $3.99\left(\mathrm{dd}, J_{5,6 \mathrm{a}} 2.2\right)$ & $3.98-4.20$ & $3.98-4.20$ & $3.99\left(\mathrm{dd}, J_{5,6 r a} 1.8\right)$ \\
\hline $\mathrm{H}-6^{\prime} \mathrm{b}$ & $\begin{array}{l}4.31\left(\mathrm{dd}, J_{5 r, 6 r \mathrm{~b}} 4.5,\right. \\
\left.J_{6 r a, 6 r b} 12.5\right)\end{array}$ & $\begin{array}{l}4.35\left(\mathrm{dd}, J_{5 r, 6 / \mathrm{b}} 4.2,\right. \\
\left.J_{6 r a, 6 r b} 12.5\right)\end{array}$ & $\begin{array}{l}4.35\left(\mathrm{dd}, J_{5 f, 6 / b}\right. \\
\left.4.2, J_{6, a, 6, b} 12.5\right)\end{array}$ & $\begin{array}{l}4.32\left(\mathrm{dd}, J_{5 r, 6 r \mathrm{~b}} 4.5\right. \\
\left.J_{6 r \mathrm{a}, 6 \circ \mathrm{b}} 12.4\right)\end{array}$ \\
\hline $\mathrm{COOCH}_{3}$ & $1.92-2.07(\mathrm{~m})$ & $1.95-2.10(\mathrm{~m})$ & $1.95-2.10(\mathrm{~m})$ & $1.94-2.06(\mathrm{~m})$ \\
\hline $\mathrm{SiC}\left(\mathrm{CH}_{3}\right)_{3}$ & & & & $0.81-0.82(\mathrm{~m})$ \\
\hline $\operatorname{Si}\left(\mathrm{CH}_{3}\right)_{2}$ & & & & $0.04-0.05(\mathrm{~m})$ \\
\hline
\end{tabular}


Table 6

${ }^{13} \mathrm{C}$ NMR chemical shifts of $2,4 \alpha, 4 \beta$, and 6

\begin{tabular}{lcccc}
\hline & 2 & $4 \boldsymbol{\alpha}$ & $\boldsymbol{4 \beta}$ & $\mathbf{6}$ \\
$\mathrm{C}-1$ & 91.54 & 89.91 & 95.12 & 95.49 \\
$\mathrm{C}-2$ & 70.38 & 71.24 & 73.18 & 73.36 \\
$\mathrm{C}-3$ & 72.31 & 69.30 & 71.95 & 72.35 \\
$\mathrm{C}-4$ & 75.79 & 76.45 & 76.45 & 77.62 \\
$\mathrm{C}-5$ & 73.48 & 68.07 & 72.86 & 72.69 \\
$\mathrm{C}-6$ & 61.57 & 61.75 & 61.75 & 62.04 \\
$\mathrm{C}-1^{\prime}$ & 100.55 & 100.58 & 100.71 & 100.74 \\
$\mathrm{C}-2^{\prime}$ & 71.50 & 71.57 & 71.57 & 71.53 \\
$\mathrm{C}-3^{\prime}$ & 72.82 & 72.92 & 72.92 & 67.84 \\
$\mathrm{C}-4^{\prime}$ & 67.81 & 67.78 & 67.78 & 71.89 \\
$\mathrm{C}-5^{\prime}$ & 71.94 & 71.83 & 71.83 & 61.52 \\
$\mathrm{C}-6^{\prime}$ & 61.57 & 61.53 & 61.53 & $169.28-170.46$ \\
$\mathrm{C}-\mathrm{COCH}$ & $169.05-170.59$ & $169.05-170.59$ & $20.49-20.72$ \\
$\mathrm{C}-\mathrm{COCH}$ & $168.72-170.37$ & $20.49-20.85$ & $20.49-20.85$ & 17.80 \\
$\mathrm{Si}-\mathrm{C}\left(\mathrm{CH}_{3}\right)_{3}$ & $20.40-20.68$ & & & 25.37 \\
$\mathrm{Si}-\mathrm{C}\left(\mathrm{CH}_{3}\right)_{3}$ & & & & -4.45 \\
$\mathrm{Si}-\left(\mathrm{CH}_{3}\right)_{2}$ & & & & -5.52 \\
& & & & \\
\hline
\end{tabular}

\section{Experimental}

Lipase AP6 (Aspergillus niger), lipase $\mathrm{P}$ (Pseudomonas sp.), lipase N (Rhizopus niveus), lipase FAP-15 (Rhizopus oryzae), lipase AY-30 (Candida cylindracea), lipase CE (Humicola sp.), lipase GC (Geotrichum candidum), lipase AK (Pseudomonas sp.), and Protease N (Bacillus sp.) (Amano Pharmaceutical. Co. Ltd, Japan), lipase OF (Candida cylindracea) and lipase MY (Candida cylindracea) (Meito-Sangyo Co. Ltd, Japan), alcalase (subtilisin Carlsberg) (Novo, Denmark), protcase II (Aspergillus oryzae), and porcine pancreas lipase (PPL) (Sigma, USA) were purchased as indicated. These crude enzymes were used for hydrolytic reactions without further purification. TLC was performed on Silica Gel G60, (E. Merck, Germany) precoated on aluminum sheets with 1:50 MeOHdiethyl ether as the developing solvent. An HPLC system (Gilson, Inc, France) used for analytical separations consisted of one 302 pump, one 305 pump, an $811 \mathrm{~B}$ dynamic mixer, an 805 manometric module, and a model 7125 syringe-loading sample injector (Rheodyne Inc, USA), coupled to a 115 variable-wavelength UV spectrophotometer and a microcomputer with HPLC method manager software (Dynamax, version 1.2) as an integrator. The two anomers were separated by using a $C_{18}$ column ( $10 \mu \mathrm{m}$ particle size, $250 \times 4.6 \mathrm{~mm}$ ) eluted with 4:1 $\mathrm{H}_{2} \mathrm{O}-\mathrm{MeCN}$ at a flow rate of $1.0 \mathrm{~mL} / \mathrm{min}$ and UV $214 \mathrm{~nm}$ detection. Optical rotations were measured with a Polartronic universal polarimeter (Schmidt and Haensch, Germany). Melting point (Büchi 510, apparatus) are uncorrected. The ${ }^{1} \mathrm{H}$ and ${ }^{13} \mathrm{C}$ NMR spectra of samples in $\mathrm{CDCl}_{3}$ were recorded with AM300 and AMX500 MHz instruments (Brüker, Germany). All chemical shifts are reported in $\mathrm{ppm}$ relative to tetramethylsilane.

$2,3,6,2^{\prime}, 3^{\prime}, 4^{\prime}, 6^{\prime}$-Hepta-O-acetyl- $\alpha, \beta$-maltose ( $3 \alpha$ and $3 \beta$ ).-To a solution of 1 ( $10 \mathrm{~g}$, $15 \mathrm{mmol})$ in either phosphate buffer $(0.1 \mathrm{M}, 20 \mathrm{~mL}, \mathrm{pH} 7$ containing $0.2 \mathrm{M} \mathrm{NaCl}$ and 3 $\left.\mathrm{mM} \mathrm{CaCl})_{2}\right)$ or the same buffer $(480 \mathrm{~mL})$ containing organic solvent $(120 \mathrm{~mL}$, shown in 
Table 1) was added the desired enzyme ( $30 \mathrm{~g})$. The mixture was stirred at $37^{\circ} \mathrm{C}$ and monitored by TLC. The reaction was stopped by extracting the products with $\mathrm{CHCl}_{3}$ and then evaporating under reduced pressure. The products, purified on a silica gel ( $70-230$ mesh) column with 4:1 hexane-diethyl ether as the eluent, showed two peaks in HPLC. From NMR identification, the major component was $2,3,6,2^{\prime}, 3^{\prime}, 4^{\prime}, 6^{\prime}$-hepta- $O$-acetyl- $\alpha$ maltose and the minor one was $2,3,6,2^{\prime}, 3^{\prime}, 4^{\prime}, 6^{\prime}$-hepta- $O$-acetyl- $\beta$-maltose; $\mathrm{mp} 176-179^{\circ} \mathrm{C}$; $[\alpha]_{\mathrm{D}}^{25}+98.0^{\circ}\left(c 1.0, \mathrm{CHCl}_{3}\right)$; lit [24] mp $179-180^{\circ} \mathrm{C},[\alpha]_{\mathrm{D}}^{15}+86.0^{\circ}\left(c 1.04, \mathrm{CHCl}_{3}\right)$. The yields and NMR data are listed in Tables 1, 3, and 4. EIMS (70 eV): $\mathrm{m} / \mathrm{z}(\%) 43(100)$, 81(10), 169(41), 211(2), 229(27), 271(1), 289(4), 331(12).

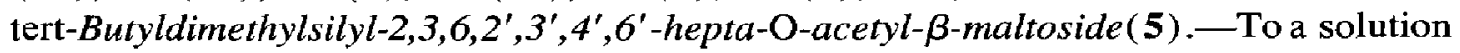
of pyridine ( $2 \mathrm{~mL}$ ), the mixture of $3 \boldsymbol{\alpha}$ and $\mathbf{3} \boldsymbol{\beta}(22 \mathrm{mg}$ ) was added. After stirring for $5 \mathrm{~min}$, tert-butyldimethylsilyl chloride was added. The mixture was stirred continuously for $50 \mathrm{~h}$, then poured into water $(20 \mathrm{~mL})$ and extracted with EtOAc $(3 \times 15 \mathrm{~mL})$. The organic extracts were evaporated and applied to an aluminum oxide column with $2: 1$ hexane-diethyl ether as eluent to give $5(23.4 \mathrm{mg}, 90 \%) ; \mathrm{mp} 101-103^{\circ} \mathrm{C} ;[\alpha]_{\mathrm{D}}^{25}+43.0^{\circ}\left(c \mathbf{1 . 0}, \mathrm{CHCl}_{3}\right)$; NMR data in Tables 3 and 4. EIMS (70 eV): $m / z(\%) 169(100), 211(6), 229(14)$, 271(5), 289(2), 331(19), 403(29), 517(25). Anal. Calcd for $\mathrm{C}_{32} \mathrm{H}_{50} \mathrm{O}_{18} \mathrm{Si}: \mathrm{C}, 51.19 ; \mathrm{H}$, 6.67. Found: C, $51.19 ; \mathrm{H}, 6.80$.

$2,3,6,2^{\prime}, 3^{\prime}, 4^{\prime}, 6^{\prime}$-Hepta-O-acetyl- $\alpha, \beta$-cellobiose ( $4 \alpha$ and $4 \beta$ ). - A solution of 2 [24] $\left(10 \mathrm{~g}, 15 \mathrm{mmol}\right.$ ) was treated as described for the preparation of $\mathbf{3 \alpha}$ and $\mathbf{3} \boldsymbol{\beta} ; \mathrm{mp} 192-198^{\circ} \mathrm{C}$; $[\alpha]_{\mathrm{D}}^{25}+27.0^{\circ}\left(c 1.0, \mathrm{CHCl}_{3}\right)$; lit $[24] \mathrm{mp} 170-190^{\circ} \mathrm{C},[\alpha]_{\mathrm{D}}^{15}+27.0^{\circ}\left(c 1.15, \mathrm{CHCl}_{3}\right)$. The yield and NMR data are in Tables 2, 5, and 6. EIMS (70 eV): $\mathrm{m} / \mathrm{z}(\%) 43(100)$, $81(10), 169(36), 211(1), 229(3), 271(1), 289(7), 331(14)$.

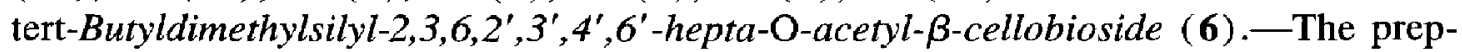
aration of 6 from the mixture of $4 \alpha$ and $4 \beta(40 \mathrm{mg})$ proceeded as described for 5 yielding $6(35 \mathrm{mg}, 75 \%)$; mp $141-143^{\circ} \mathrm{C} ;[\alpha]_{\mathrm{D}}^{25}-16.7^{\circ}\left(\mathrm{c} 2.4, \mathrm{CHCl}_{3}\right)$; NMR data in Tables 5 and 6. EIMS (70 eV): $m / z(\%) 43(100), 169(84), 211(5), 229(18), 271(5), 289(3)$, 331(29), 403(8), 517(2). Anal. Calcd for $\mathrm{C}_{32} \mathrm{H}_{50} \mathrm{O}_{18} \mathrm{Si}$ : C, 51.19; H, 6.67. Found: C, $51.26 ; \mathrm{H}, 6.87$.

\section{Acknowledgements}

We thank Miss Shou-Ling Huang for measurement of the NMR spectra at the Taipei Regional Analytical Instrumentation Center, National Science Council, Taiwan and $\mathrm{Mr}$. Fong-Ku Shi for measuring 2D ${ }^{1} \mathrm{H}-{ }^{13} \mathrm{C}$ HMQC spectra at the $\mathrm{M}$ and Vactek Corporation, Taiwan.

\section{References}

[1] J. Leroux and A.S. Perlin, Carbohydr. Res., 67 (1978) 163-178.

[2] S. Koto, N. Morishima, Y. Kihara, H. Suzuki, S. Kosugi, and S. Zen, Bull. Chem. Soc. Jpn., 56 (1983) 188191.

[3] S. Koto, N. Morishima, and S. Zen, Bull. Chem. Soc. Jpn., 52 (1979) 784-788. 
[4] J.E. Hodge and C.E. Rist, J. Am. Chem. Soc., 74 (1952) 1494-1497.

[5] W.A. Bonner, J. Am. Chem. Soc., 80 (1958) 3372-3379.

[6] A. Banaszek, X.B. Cornet, and A. Zamojski, Carbohydr. Res., 144 (1985) 342-345.

[7] A.H. Haines, Adv. Carbohydr. Chem. Biochem., 33 (1976) 11-109.

[8] R. Khan, Adv. Carbohyr. Chem. Biochem., 33 (1976) 235-294.

[9] A.L. Fink and G.W. Hay, Can. J. Biochem., 47 (1969) 353-359.

[10] M. Therisod and A.M. Klibanov, J. Am. Chem. Sac., 108 (1986) 5638-5640.

[11] H. Seino, T. Uchibori, T. Nishitani, and S. Inamasu, J. Am. Oil. Chem. Soc., 61 (1984) 1761-1765.

[12] J.F. Shaw and A.M. Klibanov, Biotechnol. Bioeng., 29 (1987) 648-651.

[13] E.W. Holla, Angew. Chem. Int. Ed. Engl., 28 (1989) 220-221.

[14] W.J. Hennen, H.M. Sweers, Y.F. Wang, and C.H. Wong, J. Org. Chem., 53 (1988) 4939-4945.

[15] M. Kloosterman, J.G.J. Weijnen, N.K. de Vries, J. Mentech, I. Caron, G. Descotes, H.E. Schocmaker, and E.M. Meijer, J. Carbohydr. Chem., 8 (1989) 693-704.

[16] S. Riva, J. Chopineau, A.P.G. Kieboom, and A.M. Klibanov, J. Am. Chem. Soc., 110 (1988) 584-589.

[17] M. Therisod and A.M. Klibanov, J. Am. Chem. Soc., 109 (1987) 3977-3981.

[18] H.M. Sweers and C.H. Wong, J. Am. Chem. Soc., 108 (1986) 6421-6422.

[19] K.Y. Chang, S.H. Wu, and K.T. Wang, J. Carbohydr. Chem., 10 (1991) 251-261.

[20] K.Y. Chang, S.H. Wu, and K.T. Wang, Carbohydr. Res., 222 (1991) 121-129.

[21] G.T. Ong, S.H. Wu, and K.T. Wang, BioMed Chem. Lett., 2 (1992) 161-164.

[22] G.T. Ong, K.Y. Chang, S.H. Wu, and K.T. Wang, Carbohydr. Res., 241 (1993) 327-333.

[23] D.G. Drueckhammer, W.J. Hennen, R.L. Pederson, C.F. Barbas, III, C.M. Gautheron, T. Krach, and C.H. Wong, Synthesis, (1991) 499-525.

[24] K. Watanabe, K. Itoh, Y. Araki, and Y. Ishido, Carbohydr. Res., 154 (1986) 165-176. 